\title{
Mitteilungen
}

\section{Ausserordentliche Ärztekammer}

Inkraftsetzung der Beschlüsse der ausserordentlichen Ärztekammer vom 11. Dezember 2008

In der statutarisch festgelegten Frist von 60 Tagen seit Publikation der Beschlüsse in der Schweizerischen Ärztezeitung (SÄZ Nr. 5 vom 28. Januar 2009) ist kein Antrag auf Urabstimmung eingereicht worden. Die Beschlüsse der ausserordentlichen Ärztekammer vom 11. Dezember 2008 sind am 29. März 2009 in Kraft getreten.

\section{Chambre médicale extraordinaire}

Entrée en vigueur des décisions de la Chambre médicale extraordinaire du 11 décembre 2008

Aucune demande de votation générale n'ayant été déposée durant le délai statutaire des 60 jours après la publication des décisions de la Chambre médicale extraordinaire du 11 décembre 2008 dans le Bulletin des médecins suisses (BMS n ${ }^{\circ} 5$ du 28 janvier 2009), elles sont entrées en vigueur le 29 mars 2009.

\section{Prix Perspectives 2009}

\section{Förderpreis für Engagement in den Bereichen Schizophrenie und Bipolare Störungen}

Der Prix Perspectives hat es sich zur Aufgabe gemacht, einen Beitrag zum Wohle von schizophren und bipolar Erkrankten zu leisten. Mit 25000 Franken werden Schweizer Projekte ausgezeichnet, die sich in den Bereichen Schizophrenie oder Bipolare Störungen engagieren - ob Selbsthilfegruppen, Betreuungsangebote oder Kontaktstellen für Angehörige. Bewerben können sich sowohl Privatpersonen, organisierte Gruppen als auch Kliniken. Einsendeschluss ist der 29. Mai 2009. Eine Experten-Jury entscheidet anschliessend über die Vergabe des Preises.

Kontakt und weitere Informationen: antwerpes ag, Christiane Schrix, Vogelsanger Strasse 66 D-50823 Köln, Tel. +49 22192053 319, Fax +49 22192053 133, E-Mail: christiane.schrix@ antwerpes.de

\section{AQUIRA Awards}

Die Schweizer Niederlassung des US-Pharmaunternehmens Bristol-Myers Squibb setzt sich für innovative Therapien im Bereich der Rheumatoiden Arthritis und anderer Autoimmunerkrankungen ein. Um einen Beitrag zur Qualitätsverbesserung zu leisten, hat Bristol-Myers Squibb die AQUIRA Awards entwickelt. AQUIRA steht dabei für Awards for QUality management In Rheumatoid arthritis and other Autoimmune diseases. In der Schweiz niedergelassene Spital- und Praxisärzte können Projekte einreichen. Die von einer hochrangigen Jury ausgewählten Projekte werden mit Beiträgen von insgesamt 70000 Franken bei ihrer Realisierung unterstützt. Eingabeschluss ist der 1. Juni 2009. Die Jury wird präsidiert von Prof. Dr. Peter M. Villiger.

Informationen: www.aquira.ch

La succursale suisse du laboratoire pharmaceutique américain Bristol-Myers Squibb s'engage en faveur des thérapies novatrices dans le domaine de la polyarthrite rhumatoïde et d'autres maladies auto-immunes. BristolMyers Squibb a conçu les AQUIRA Awards pour contribuer à l'amélioration de la qualité lors de la prise en charge de ces maladies. AQUIRA est l'acronyme d'Awards for QUality management In Rheumatoid arthritis and other Autoimmune diseases. Les médecins exerçant en Suisse à l'hôpital ou en cabinets privés peuvent adresser des projets. Les projets élus par un jury de haut rang seront soutenus dans leur réalisation par des subventions d'un montant total de 70000 francs. La date limite de dépôt des dossiers est fixée au $\mathbf{1}^{\text {er }}$ juin 2009. Le jury sera vice-présidé par le professeur Alexander So du CHUV à Lausanne.

Informations: www.aquira.ch

\section{KIKOM}

[foif $] \mathrm{x}[$ eis] Schweizerischer Förderpreis für Komplementärmedizin 2009

Fachgebiete: Traditionelle Chinesische Medizin, Aurikulomedizin und Klassische Homöopathie

Arbeiten/Kriterien: Abgeschlossene wissenschaftliche Arbeiten, Lehrbücher oder Skripten von Autoren aus der Schweiz oder von im Ausland tätigen Schweizern. Es können eine oder mehrere Arbeiten ausgezeichnet werden. Bei der Preisvergabe an mehrere Arbeiten wird die Preissumme aufgeteilt. Der Rechtsweg ist ausgeschlossen.

Preissumme: jährlich 11111 [foif]x[eis] Franken

Preisverleihung: 3. ASA-TCM-Kongress in Basel vom 3./4. Dezember 2009

Eingabetermin: 31. Juli 2009

Information: Teilnahmebedingungen sind bei foifmaleis@kikom.unibe.ch erhältlich

[cinq]x[un] Prix suisse d'encouragement pour la médecine complémentaire 2009

Spécialités: médecine traditionnelle chinoise, auriculomédecine, homéopathie classique uniciste.

Travaux/critères: travaux scientifiques conclus, manuels ou traités présentés par des auteurs travaillant en Suisse ou par des Suisses à l'étranger. Le prix pourra être discerné à un ou plusieurs travaux. Dans le second cas, la somme du prix sera partagée. Aucun recours n'est possible.

Montant du prix: 11111 [cinq]x[un] francs par année.

Distribution du prix: lors du $3^{\mathrm{e}}$ congrès ASATCM à Bâle les 3 et 4 décembre 2009.

Délai de présentation: 31 juillet 2009

Informations: les conditions de participation peuvent être obtenues sous: foifmaleis@kikom. unibe.ch 
DRG-Symposium

\section{Jahre DRGs in Deutschland - Lehren für die Schweiz}

Am Montag, 11. Mai 2009, findet im Zürcher Mariott Hotel von 9.00 bis 17.30 Uhr ein Symposium zur Einführung der DRG (diagnosisrelated groups $=$ Fallpauschalen) in der Schweiz statt, in dessen Rahmen namhafte Experten referieren und die Thematik unter Einbezug des Publikums diskutieren.

Die Vorarbeiten zur Umsetzung der SwissDRG sind in vollem Gange. Die nun beschlossenen KVG-Verordnungen haben weitere Klärungen gebracht, viele Fragen sind aber noch offen. Als «Nachzüglerin» kann und sollte die Schweiz von ausländischen Erfahrungen profitieren. Nachdem beschlossen worden ist, das deutsche DRG-System zu übernehmen und zu helvetisieren, liegt es nahe, insbesondere von den dortigen Erfahrungen zu lernen. Kompetente Referenten und Referentinnen beleuchten das Thema aus der Sicht ihres eigenen Aufgabenbereichs. Für die Einführung des DRG-Systems in der Schweiz ist eine interdisziplinäre Arbeitsweise der verschiedenen Akteure unbedingt notwendig.

Ziel des Symposiums: Aus der Praxis für die Praxis: Es wird besonderer Wert darauf gelegt, den Teilnehmenden umsetzbare Empfehlungen zu präsentieren (Diskussions- und Fragerunden mit den Referenten und dem Podiumsgast PD Dr. Simon Hölzer).

Zielpublikum: Verantwortungsträger und Akteure aus allen Bereichen des schweizerischen Gesundheitswesens, Spitäler, Krankenversicherer, Behördenvertreter von Bund und Kantonen, Pharma- und Medizinprodukteindustrie, Unternehmensberatungen, am Gesundheitswesen interessierte Politikerinnen und Politiker, Medienschaffende und Verbände.

Moderation: Dr. rer. pol. Heinz Locher, Management + Consulting Services, Bern.

Referenten: Dr. iur. Carlo Conti, Verwaltungsratspräsident der SwissDRG AG, Vorsteher des Gesundheitsdepartements Basel-Stadt; Dr. phil. nat. Bernhard Wegmüller, Direktor H+ Die Spitäler der Schweiz; Otto Bitterli, CEO Sanitas Krankenversicherung und Mitglied des Verwaltungsrats von santésuisse; Prof. Dr. med.
Marc A. Reymond, MBA (AACSB), Schweizer Chirurg und Viszeralchirurg, seit 2005 Chefarzt und Budgetverantwortlicher der Klinik für Allgemein-, Viszeral- und Thoraxchirurgie am Evangelischen Krankenhaus Bielefeld; Prof. Dr. med. Burkhard Göke, seit 2007 Ärztlicher Direktor des Klinikums der Ludwig-Maximilians-Universität München, davor Ordinarius und Chefarzt für Gastroenterologie, Inselspital Bern; Dr. Pierre-François Cuénoud, Chefarzt (Chirurgie) im Spital Sion, Mitglied des Zentralvorstandes der FMH, Leiter Ressort Swiss DRG und Vorstand H+; Markus Wittwer, Direktor Pflege und Personal, stellvertretender Spitaldirektor, Kantonsspital Winterthur, Vorstandsmitglied Schweizerische Vereinigung der Pflegedienstleitungen (SVPL), MAS Health Service Management.

Podiumsgast: PD Dr. Simon Hölzer, Geschäftsführer SwissDRG AG.

Weitere Informationen und Anmeldung: Unter www.rsmedicalconsult.com. Teilnahmegebühr 590 Euro inkl. Mittagessen. Die Teilnehmerzahl ist begrenzt! Die Themenschwerpunkte werden ständig an den neuesten Sachstand angepasst, so dass etwaige Programmänderungen vorbehalten bleiben.

\section{med-swiss.net}

Managed Care statt Kontrahierungsstreit! Faire Rahmenbedingungen für Ärztenetzwerke

Wie soll Managed Care (MC) gefördert werden, um zu einer mehrheitsfähigen Lösung im $\mathrm{Zu}$ sammenhang mit den Themen Ärztezulassung und Tarifvertrag beizutragen? Zu dieser Frage veranstaltet der Dachverband der Schweizer Ärztenetzwerke med-swiss.net eine Podiumsdiskussion.

Bewusste und gezielte Steuerung im Gesundheitswesen ist sinnvoll und zunehmend notwendig. Weniger eindeutig sind die Antworten auf Fragen, wer wen oder was steuert und mit welchen Instrumenten und Zielen.

Die im Dachverband med-swiss.net vereinigten Ärztenetzwerke haben diesbezüglich klare Vorstellungen, denn die ihnen angeschlossenen Ärztinnen und Ärzte leben MC tagtäglich, zusammen mit ihren Patientinnen und Patienten sowie deren Krankenversicherern.
MC darf keinesfalls als Prämiensparmodell verstanden und behandelt werden. MC soll im KVG mit einem eigenen, positiv ausformulierten Artikel umschrieben werden. Zentrale Elemente sind dabei

- der MC-Vertrag zwischen Versicherern und Netzwerken bezüglich Umfang, Preis und Qualität der zu erbringenden Leistungen;

- der reduzierte Selbstbehalt für MC-Patienten;

- die Freiwilligkeit aller Beteiligten, an einem MC-Modell zu partizipieren.

Den genauen Wortlaut unseres Vorschlages eines neuen MC-Artikels finden Sie auf unserer Website www.med-swiss.net.

Budgetmitverantwortliche Ärztenetzwerke bieten als einzige Versorgungsform in der Schweiz einen Ausgleich zwischen Überversorgung und Unterversorgung an, indem sie die Einzelleistungsverrechnung mit der Budgetpflicht kombinieren.

Als Podiumsteilnehmer konnten SR Christine Egerszegi, FDP AG, NR Ruth Humbel, CVP AG, NR Dr. med. Ignazio Cassis, FDP TI, Vizepräsident FMH, PD Dr. Konstantin Beck, CSSVersicherungen, Dr. med. Felix Huber, Präsident Verwaltungsrat mediX Zürich AG, und Dr. med. Alfred Rösli, Präsident Verwaltungsrat hawadoc AG, Winterthur, gewonnen werden. Diskussionsleiterin: Claudia Schoch, Inlandredaktorin der NZZ.

Wenn Sie am Schicksal des Schweizer Gesundheitswesens, an Lösungsvorschlägen im Zusammenhang mit den anstehenden Finanzierungsproblemen und den daraus hervorgehenden Managed-Care-Fragen interessiert sind, so reservieren Sie sich den nachgenannten Ter$\min$.

Datum: Donnerstag, 14. Mai 2009, 16.1518.30 Uhr, anschliessend Apéro

Ort: Zunfthaus zur Schneidern (Blue Monkey), Stüssihofstatt 3, 8001 Zürich

Anmeldungen bitte per Mail, Fax oder Post an: med-swiss.net, Grütlistrasse 36, 8002 Zürich, Fax 04428044 03, E-Mail: info@med-swiss.net. Wir senden Ihnen die Anmeldebestätigung gerne an Ihre E-Mail-Adresse. 